\title{
Comparación de la actividad antihipertensiva de fosinopril e irbesartan
}

\author{
E. ANGULO, N. R. ROBLES*, J. GROIS**, A. BARQUERO***, M. PÉREZ \\ MIRANDA**** \\ Servicio de Medicina Interna, *Unidad de Hipertensión Arterial, **Servicio de \\ Hematología y *** Análisis Clínicos. Centro de Salud Anexo I. Hospital Infanta Cristina. \\ Badajoz
}

\author{
COMPARATION OF ANTIHYPERTENSIVA ACTIVITY OF FOSINO - \\ PRIL AND IRBESARTAN
}

\section{RESUMEN}

Objetivo: Se ha comparado la eficacia antihipertensiva de dos fármacos de administración única diaria que actúan por mecanismos diferentes sobre el eje renina-angiotensina.

Método: Se incluyeron en el estudio 30 pacientes seguidos durante 3 meses. Quince fueron tratados con el IECA fosinopril (grupo F), estos pacientes tenían una edad media de 57,4 $\pm 11,5$ años, siendo 9 hombres y 6 mujeres. Otros quince pacientes fueron tratados con el antagonista de receptores de angiotensina irbesartan (grupo I), tenían una edad media de $65,2 \pm 8,7$ años, siendo 4 hombres y 9 mujeres. Las diferencias en edad y sexo entre ambos grupos no fueron significativas. Tras confirmar la presencia de HTA leve-moderada los pacientes fueron seleccionados de forma aleatoria para iniciar tratamiento con fosinopril $(20 \mathrm{mg})$ o irbesartan $(150 \mathrm{mg} / \mathrm{día})$. En los casos en que la respuesta no fué suficiente, se añadieron $12,5 \mathrm{mg}$. de hidroclorotiazida al tratamiento.

Resultados: La PAS inicial fue 147,9 $\pm 11,7$ en el grupo F y 157,7 \pm $11,1 \mathrm{mmHg}$ en grupo I $(\mathrm{p}<0,05)$ y la PAD fue respectivamente $92,3 \pm$ 6,29 y $94,1 \pm 5,6 \mathrm{mmHg}$ (diferencia no significativa). Tras tres meses de tratamiento las PAS descendió a 132,3 $\pm 12,4$ (grupo $F, p=0,015$ ) y $131,0 \pm 8,7$ (grupo I, $p=0,002$ ). La PAD disminuyó a 84,1 $\pm 5,41$ (grupo $\mathrm{F}, \mathrm{p}=0,001$ ) y $82,7 \pm 4,16$, (grupo $\mathrm{I}, \mathrm{p}=0,001$ ). Las diferencias entre las medias finales ambos grupos no son significativas. Sin embargo, el descenso de la PAS en el grupo I $(26,7 \pm 11,6 \mathrm{mmHg})$ fue significativamente superior a la obtenida en el grupo $\mathrm{E}(15,6 \pm 11,6 \mathrm{mmHg}, \mathrm{p}=0,011)$. En el grupo $\mathrm{F}$ el descenso de la PA fué significativo desde el primer mes (PAS 140,7 $\pm 12,2, p=0,021 ; \operatorname{PAD} 87,2 \pm 6,2, p=0,003$ ). En el grupo I el descenso sólo fué significativo a partir del segundo mes (PAS 135,5 \pm $10,4, \mathrm{p}<0,001 ; \operatorname{PAD} 85,3 \pm 4,3, \mathrm{p}<0,001)$.

Conclusiones: Tanto fosinopril como irbesartan parecen igualmente eficaces para reducir la PAD. El irbesartan parece tener mayor eficacia sobre la presión arterial sistólica. El efecto del irbesartan se realiza de forma más gradual evitando caidas bruscas de la PA.

PALABRAS CLAVE: Hipertensión arterial. Tratamiento. Eje reninaangiotensina.

\section{ABSTRACT}

Objective: To compare the antihypertensive effect of two daily single dose drugs acting on the renin-angiotensin axis by two different ways.

Methods: Thirty patients were randomized to receive either irbesar tan (150 mg once daily) ( $n=15$, mean age $65.2 \pm 8.7$ years, 9 men and 6 women) or fosinopril ( $20 \mathrm{mg}$ once daily) ( $n=15$, mean age $57.4 \pm 11.5$ years, 4 men and 11 women, difference are not significant) during 12 weeks. When needed, hydroclorothiazide $(12,5 \mathrm{mg})$ was added to treat ment to improve hypotensive response.

Results: A reduction of $S B P$ and $D B P$ was observed in bothl treat ment groups throughout the study. In order to obtain further BP reduction, hydrochlorothiazide was added to 6 patients with inadequate BP response at the 4th week (3 patients in the irbesartan group) and 8th week (2 patients in irbesartan group and 1 patient in fosinopril group). $S B P$ was reduced in irbesartan group from $157.7 \pm 11.2$ to $131.0 \pm 8.7$ $\mathrm{mmHg}(12$ th week, $p<0.001)$. DBP decreases from $94.1 \pm 5.6$ to $82.7 \pm$ $4.2 \mathrm{mmHg}(p<0.001)$. In fosinopril group SBP was reduced from 147.9 \pm 11.7 to $132.2 \pm 12.4 \mathrm{mmHg}(p<0.001)$ and $\mathrm{DBP}$ decreases from $92.3 \pm 6.3$ to $84.0 \pm 5.4 \mathrm{mmHg}(p<0.001)$. Final between group differences in BP are not significant. Final BP reduction in irbesartan group $(26.7 \pm 11.6 \mathrm{mmHg})$ was bigger than that obtained in fosinopril group $(15.6 \pm 11.6 \mathrm{mmHg}, p=0.011)$. BP reduction was significant in fosinopril group from the first month (SBP $140.7 \pm 12.2, p=0,021 ; D B P 87.2 \pm 6.2$, $p=0.003)$. In irbesartan group BP reduction was not significant until the second month (SBP 135.5 $\pm 10.4, p<0.001 ; D B P 85.3 \pm 4.3, p<0.001)$.

Conclusions: Fosinopril and irbesartan seems to be equally efective to reduce DBP. Irbesartan might have higher effectiveness on systolic blood pressure. Irbesartan act more gradually than fosinopril and this may be useful to prevent from acute blood pressure falls.

KEY WORDS: Hypertension. Treatment. Renin-angiotensin axis.

Angulo E, Robles NR, Grois J, Barquero A, Pérez Miranda M. Comparación de la actividad antihipertensiva de fosinopril e irbesartan. An Med Interna (Madrid) 2002; 19: 571-575.

Trabajo aceptado: 10 de julio de 2002

Correspondencia: Nicolás Roberto Robles. Unidad de HTA. Hospital Infanta Cristina. Ctra. de Portugal, s/n. 06070 Badajoz. e-mail: nroblesp@meditex.es 


\section{INTRODUCCIÓN}

La introducción en los años 80 de los inhibidores de la enzima conversora de la angiotensina (IECA) en la farmacopea significó un avance revolucionario en el tratamiento de la hipertensión arterial y de sus complicaciones asociadas (1). Aunque los fármacos que actuan antagonizando los receptores de la angiotensina (ARA) son más antiguos que estos, hasta años recientes no han podido ser utilizados de forma eficaz en el tratamiento crónico de la HTA (2).

Además de sus efectos como antihipertensivo, que incluyen la reducción de la morbimortalidad asociada a la HTA, los IECA han demostrado otras acciones específicas relacionadas en buena parte con su capacidad para impedir las acciones de la angiotensina (3-6). Pese a su reciente introducción, los ARA, siguiendo el camino abierto por los IECA, han demostrado ser capaces de producir estos mismos efectos beneficiosos en algunos campos como la insuficiencia cardiaca congestiva o la nefropatia diabética (7-9).

Se han realizado diversas comparaciones terapeúticas de los ARA con otros fármacos antihipertensivos $\mathrm{y}$, particularmente, con IECA, especialmente captopril y enalapril. Estos dos fármacos, si bien muy utilizados para el tratamiento de la hipertensión en todo el mundo, poseen dos características que les diferencian de otros IECA, corta vida media y escasa fijación tisular (10). Hemos creido interesante comparar el efecto de un ARA con larga vida media, irbesartan (11), con un IECA, fosinopril, que posee tambien vida media prolongada (10), tanto en cuanto a su eficacia antihipertensiva como a sus efectos colaterales.

\section{MATERIAL Y MÉTODOS}

Participaron en el estudio 30 pacientes con HTA leve o moderada según los criterios de la WHO-ISH. Se excluyeron aquellos pacientes con historia de intolerancia a IECA o ARA, mujeres embarazadas, pacientes con insuficiencia renal (definida como una creatinina sérica superior a $1,5 \mathrm{mg} / \mathrm{dl}$ ) o hiperpotasemia y aquellos que hubieran padecido un acontecimiento cardiovascular grave en los seis meses previos a su incorporación en el estudio. En el caso de que el paciente recibiera tratamiento con otro fármaco antihipertensivo, este fué suspendido 15 días antés de iniciar el nuevo tratamiento.

Los pacientes fueron distribuidos aleatoriamente para recibir tratamiento con fosinopril o irbesartan. Las características de ambos grupos han sido recogidas en la tabla I. Los pacientes del grupo I resultaron tener mayor edad y mayor PAS que los enfermos del grupo F. En cuanto a otros factores de riesgo, el grupo I incluía tambien 6 enfermos diabéticos, mientras que no habia ninguno en el grupo F. La distribución de otros factores de riesgo cardiovascular no mostraba ninguna diferencia entre ambos grupos (Fig. 1).

Los enfermos fueron tratados inicialmente con fosinopril $(20 \mathrm{mg})$ o irbesartan $(150 \mathrm{mg})$ en dosis única diaria administrada por la mañana. En este momento se extrajo sangre para determinaciones bioquímicas y hemograma. Los enfermos fueron citados al més y reevaluados. Si el paciente presentaba PA elevada pese al tratamiento antihipertensivo, se añadió hidroclorotiazida, $12,5 \mathrm{mg} /$ día tomados junto con el fármaco principal. Los enfermos eran citados de nuevo en 1 mes (sesenta días desde el comienzo del tratamiento) para valoración clínica (sin

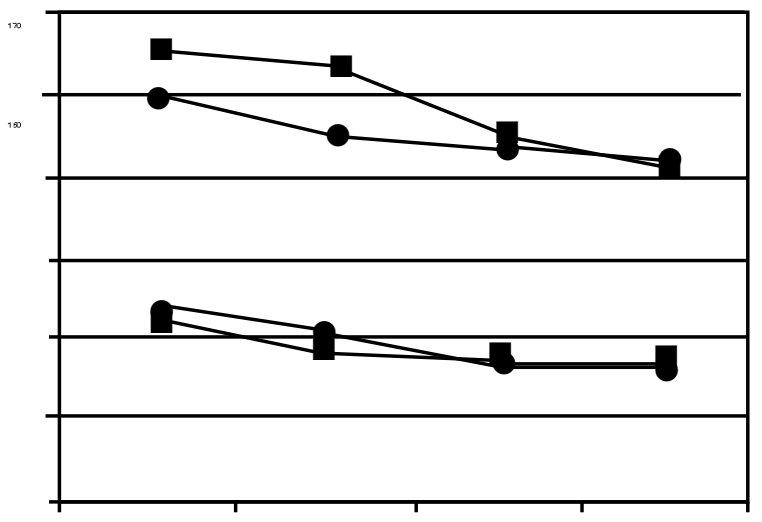

Fig. 1. La PAS era inicialmente más alta en el grupo tratado con irbesartan. La reducción de la PAS fué más lenta en el grupo tratado con irbesartan, pero al final del estudio, era menor que en el grupo tratado con fosinopril. La reducción de la PAD fue similar en ambos grupos. Significaciones en el texto.

análisis) y en el caso de que no se hubieran controlado trás la adición del diurético eran excluidos del estudio. Aquellos que mostraran mal control con un sólo fármaco recibían tambien diuréticos en la misma dosis. Se realizó una última visita de valoración a los 90 días de iniciar tratamiento, en la que se incluyo exploración física y determinaciones analíticas. Los estudios analíticos se realizaron según técnicas estandarizadas.

Las comparaciones estadísticas se han realizado utilizando el programa estadístico SPSS. Los datos de variables continuas se han comparado mediante la prueba de la "t" de Student para datos relacionados o no relacionados según fueran medias del mismo grupo de pacientes o del grupo con el que se realizaba la comparación. Los datos de frecuencia se han comparado mediante la prueba de chi cuadrado o la prueba exacta de Fischer (si en alguna de las casillas habia menos de 5 casos). Los valores se han expresado como media \pm 1 desviación típica.

\section{RESULTADOS}

La PAS media inicial en el grupo F fue de 147,93 \pm 11.71 mmHg y la PAD 92,33 $\pm+6,29 \mathrm{mmHg}$ Al final del estudio la PAS habia descendido hasta $132,33 \pm 12,37 \mathrm{mmHg}(\mathrm{p}<0,001)$. y la PAD a 84,0 $\pm+5,41 \mathrm{mmHg}(\mathrm{p}<0,001)$ (Fig. 1, Tabla I). El número de pacientes que normalizaron su PAS $(<140 \mathrm{mmHg})$ al final del tratamiento fue 11 pacientes $(73 \%)$. La PAD descendió por debajo de $90 \mathrm{mmHg}$ en 13 pacientes (87\%) (p $=0,02$ ) (Fig. 2). Dos pacientes (en la tercera y cuarta visita respectivamente) necesitaron añadir diurético al tratamiento.

Los pacientes tratados con Irbesartan presentaban unos valores medios iniciales de PAS de $157,66 \pm+11,15 \mathrm{mmHg}$ ( $p<0,05$ respecto a los pacientes del grupo F). La PAS sufrió un descenso lento al inicio, que no fue significativo al mes $(154,3+16,2 \mathrm{mmHg}$, ) pero si a partir del segundo mes el descenso alcanzo significación estadística $(136,5 \pm 10,4$ $\mathrm{mmHg}, \mathrm{p}<0,01)$. Al final del tratamiento la PAS media se había reducido hasta $131,0 \pm 8,7 \mathrm{mmHg}(\mathrm{p}<0,001)$ (Fig. 1, Tabla I). 
TABLA I

EVOLUCIÓN DE LA PA EN AM BOS GRUPOSDE TRATAM IENTO

\begin{tabular}{lcccc}
\hline & Visita 0 & Visita 1 & Visita 2 & Visita 3 \\
\hline PAS Irbesartan & $157,7 \pm 11,6$ & $154,3 \pm 16,2$ & $136,5 \pm 10,4$ & $131,0 \pm 8,7$ \\
PAS Fosinopril & $147,9 \pm 11,7$ & $140,7 \pm 12,2$ & $135,0 \pm 11,2$ & $132,2 \pm 12,4$ \\
PAD Irbesartan & $94,1 \pm 5,6$ & $90,7 \pm 7,5$ & $85,3 \pm 4,4$ & $82,7 \pm 4,2$ \\
PAF Fosinopril & $92,3 \pm 6,3$ & $87,2 \pm 6,2$ & $85,5 \pm 5,0$ & $84,01 \pm 5,4$ \\
\hline
\end{tabular}

Doce pacientes normalizaron su PAS $(80 \%$, no es significativo frente a grupo F). La PAD mostró un descenso progresivo (inicial, 94,1 \pm 5,6; final de $82,7 \pm 4,2 \mathrm{mmHg}, \mathrm{p}<$ $0,001)$. Todos los pacientes normalizaron su PAD al final del tratamiento $(100 \%$, la diferencia no es significativa frente a $\mathrm{F})$ (Fig. 2). Seis pacientes necesitaron añadir diurético al tratamiento, tres en el segundo control y los tres restantes en el tercer y cuarto control. La diferencia en el número de pacientes que precisaron diurético no es estadisticamente significativa.

Hubo un descenso mayor de la PAS en los tratados con Irbesartan $(26,7 \pm 11,6 \mathrm{mmHg})$ que en los tratados con fosinopril $(15,6 \pm 10,6 \mathrm{mmHg}, \mathrm{p}<0,01)$. Lo mismo ocurrió con la PAD que se redujo significativamente más con irbesartan $(11,5 \pm 5,1 \mathrm{mmHg})$ que con fosinopril $(8,3 \pm 4,4 \mathrm{mmHg}, \mathrm{p}<$ 0,05) (Fig. 2).

Para valorar mejor el efecto de cada fármaco se han repetido las comparaciones excluyendo a los pacientes que habían recibido tratamiento con diuréticos. El estudio comparativo entre ambos grupos terapéuticos sin hidroclorotiacida, mostró diferencias significativas en la PAS al inicio del tratamiento $(\mathrm{p}<0,05)$, pero esta diferencia no alcanzaba significación en visitas posteriores. En la PAD no hubo diferencias significativas desde el inicio al final del tratamiento entre ambos grupos (Tabla II). El descenso final fue significativamente mayor en
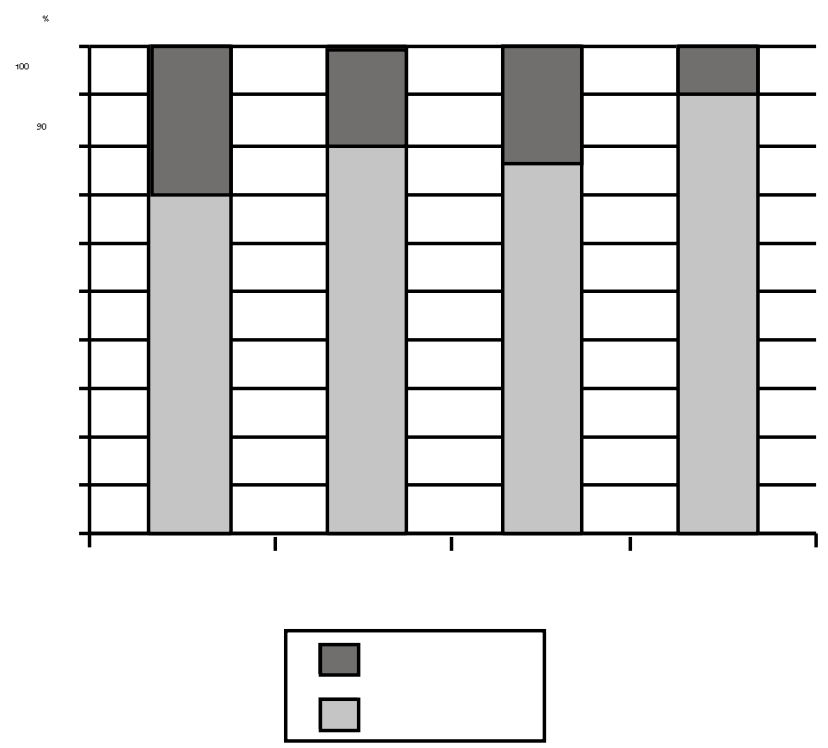

Fig. 2. El número de pacientes controlados al final del estudio fue similar en ambos grupos.
TABLA ॥

EVOLUCIÓN DE LA PA EN LOS PACIENTES Q UE NO FUERON TRATADOS CON DIURÉTICOS

\begin{tabular}{lcccc}
\hline & Visita 0 & Visita 1 & Visita 2 & Visita 3 \\
\hline PAS Irbesartan & $156,7 \pm 12,2$ & $146,1 \pm 14,5$ & $132,0 \pm 8,4$ & $127,8 \pm 17,1$ \\
PAS Fosinopril & $144,9 \pm 10,9$ & $138,1 \pm 10,9$ & $132,3 \pm 7,8$ & $130,8 \pm 12,2$ \\
PAD Irbesartan & $94,7 \pm 6,6$ & $86,7 \pm 6,6$ & $83,9 \pm 3,3$ & $81,1 \pm 3,3$ \\
PAF Fosinopril & $92,3 \pm 6,1$ & $86,4 \pm 5,5$ & $84,41 \pm 4,4$ & $84,21 \pm 5,7$ \\
\hline
\end{tabular}

el grupo tratado con irbesartan (sin diurético) que en el grupo tratado con fosinopril (sin diurético) tanto para la PAS $(28,9 \pm 11,4$ frente a $14,2 \pm 10,7 \mathrm{mmHg}$ con fosinopril, $\mathrm{p}<0,01)$ como para la PAD (irbesartan 13,6 $\pm 5,4$ frente a fosinopril $8,1 \pm 4,2$, p $<0,01$ ) (Fig. 3).

No hubo efectos secundarios clínicamente detectables. No se detectó ningún caso de hiperkaliemia ni de insuficiencia renal. Ningún paciente debió interrumpir tratamiento debido a efectos indeseables.
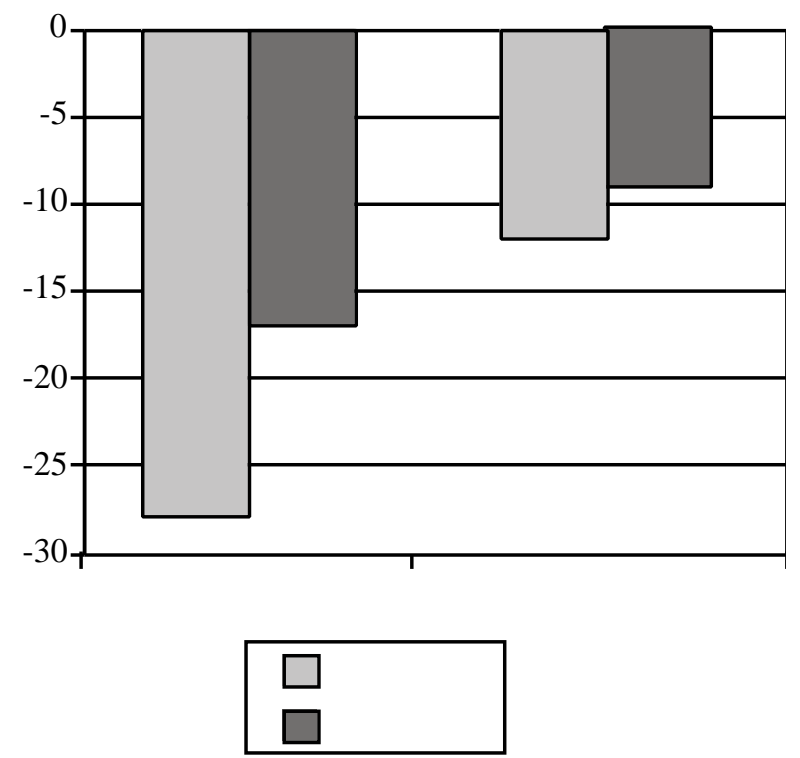

Fig. 3. La reducción de la PA tanto sistólica como diastólica seguía siendo superior en el grupo tratado con irbesartan una vez excluidos los enfermos en los que hubo que asociar un diurético al tratamien. to.

\section{DISCUSIÓN}

A primera vista, fosinopril e irbesartan fueron igualmente eficaces en la reducción de la presión arterial. Existen varias comparaciones en la bibliografia que muestran resultados semejantes, tanto con irbesartan, como con otros antagonistas de receptores de la angiotensina (12-15). Sin embargo, por efecto de la randomización necesaria en el reclutamiento de los pacientes, los grupos no fueron totalmente homogéneos, y el grupo tratado con irbesartan tenía una edad levemente superior al grupo tratado con fosinopril. De la misma manera, se reclutaron 6 diabéticos y todos ellos estaban en el grupo trata- 
do con irbesartan. Ambas condiciones, edad avanzada y diabetes mellitus se asocian a una mayor incidencia de hipertensión arterial sistólica, como es bien conocido $(16,17)$. La consecuencia final es que la PAS del grupo tratado con irbesartan era superior a la del grupo tratado con fosinopril. Este fenomeno es difícil de evitar, incluso en estudios que incluyen gran número de enfermos, como en el Captopril Prevencion Project (CAPPP), donde el grupo tratado con captopril tenía una PAS al inicio superior al grupo que no recibió este fármaco (6).

Cuando se examina la respuesta de la PAS al tratamiento puede observarse una diferencia clara entre ambos grupos. En el grupo que recibió fosinopril hay una reducción de la PAS desde la primera visita al més de comienzo. En el grupo en tratamiento con irbesartan la respuesta fue más lenta, no siendo significativa hasta la segunda visita, a los 60 días. Esta diferencia no es sorprendente. Si bién el efecto de la mayor parte de los agentes antihipertensivos suele ser máxima a las 4 semanas de su uso, los antagonistas de receptores de la angiotensina ejercen su acción hacia las 6 semanas (18-21). Es decir, su comienzo de acción es gradual, lo que puede ser un beneficio sustancial cuando no existen motivos para tener prisa en la reducción de la presión arterial, como ocurre en las hipertensiones leves, y un inconveniente si el paciente precisa un tratamiento rápido y potente por la presencia de cifras muy elevadas o complicaciones asociadas.

Es interesante también, observar que la PAS con irbesartan era inferior al final del periodo de observación, aunque esta diferencia no fuera significativa. Sin embargo, si era significativamente mayor la reducción de la PAS obtenida con irbesartan respecto a la conseguida con fosinopril, es decir, la diferencia entre presiones iniciales y presiones finales. Puesto que el grupo con irbesartan precisó añadir al tratamiento diurético en un mayor número de pacientes, podría pensarse que este mayor efecto del irbesartan es ocasionado por el uso del diurético. Para eliminar este posible sesgo se han vuelto a realizar las comparaciones estadísticas excluyendo los enfermos de cada grupo terapeútico. El resultado, no varía, la reducción final de la PAS obtenida con irbesartan sigue siendo superior que la obtenida con fosinopril. Este efecto parece concordar con los resultados obtenidos con otro ARA, el eprosartan, el cual, en comparación con enalapril, reduce de forma más intensa la PAS (22). Esta acción podría estar relacionada con un mayor efecto terapeútico como bloqueante de la angiotensina, puesto que la administración de IECA produce no sólo un descenso de la PA sino, también, un aumento de la distensibilidad de la pared arterial que es independiente de la reducción de presión y no se asocia a ningún cambio en la presión tangencial que soporta la pared, excluyendo la interacción de factores mecánicos en esta acción beneficiosa $(23,24)$. No obstante, no podemos excluir la existencia de un fenomeno de regresión a la media desde valores de PA basales más elevados.
En cuanto a la PAD, esta no era diferente en principio entre ambos grupos, a diferencia de la PAS, y se redujo de forma similar con ambos tratamientos, ocurriendo un cruce similar: La PAD era ligeramente superior en los grupo I al comenzar el seguimiento y se habia reducido por debajo de la PAD de los enfermos que recibían fosinopril al final del tratamiento, sin alcanzar significación estadística. Una vez más, la reducción final de la PAD, la diferencia entre las PAD iniciales y finales, fue significativamente superior al final del estudio, incluso cuando se excluye a los enfermos que necesitaron diuréticos para alcanzar el nivel de presión arterial deseado.

Un número mayor de enfermos precisaron añadir un diurético en el grupo I, pero esta diferencia no era significativa. De todas formas, en la mayor parte de los estudios poblacionales, un porcentaje superior al $40 \%$ de los pacientes necesitan añadir un segundo fármaco hipotensor, proporción que no se alcanzó en ninguna de las dos ramas del estudio.

Estos resultados sugieren una mayor efectividad de los fármacos antagonistas de los receptores de la angiotensina para reducir la PA, sistólica o diastólica, a largo plazo, aunque su acción sea más gradual y su efecto no pudiera detectarse en las primeras visitas. Este efecto ha podido verse en algunas experiencias previamente publicadas, pero no es un hallazgo universal (22). En particular, los dos estudios previamente publicados comparando irbesartan y otro inhibidor de la ECA, el enalapril, no encontraron diferencias entre ambos $(14,15)$.

Las razones de esta mayor efectividad de los antagonistas de receptores de la angiotensina deben buscarse sin duda en su mecanismo de acción: bloqueo completo del receptor tipo I de la angiotensina y con ello bloqueo del eje renina-angiotensina sin que su efecto pueda ser compensada por las vias alternativas de producción de angiotensina II. Por otra parte, la angiotensina producida por la enzima conversora que no puede alcanzar los receptores tipo I de la angiotensina si puede estimular los receptores tipo II de la angiotensina, que producen efecto vasodilatador e hipotensor, puesto que no son bloqueados por estos fármacos $(2,25)$.

En cualquier caso, la conclusión final con nuestra experiencia es que los agentes antagonistas de los receptores tipo I de la angiotensina II son activos para reducir ambos componentes de la presión arterial y podrían ser más eficaces que los clásicos inhibidores de la enzima conversora de la angiotensina. No obstante, dado el limitado tamaño de la muestra utilizada serán precisos estudios más amplios para llegar a una conclusión definitiva. El efecto hipotensor de los antagonistas de los receptores de la angiotensina se produce de una forma más gradual, lo cual puede ser una propiedad de gran interés en las hipertensiones leves. No obstante, es conveniente ampliar el periodo de evaluación de la eficacia del tratamiento con estos fármacos hasta las 6 semanas de tratamiento, en lugar de las 4 habitualmente recomendadas con otros hipotensores.

\section{Bibliografía}

1. Cushman DW, Cheung HS, Sabo EF, Ondetti MA. Development and design of specific inhibitors of angiotensin-converting enzyme. Am J Cardiol 1982; 40: 1390-1394.

2. Goodfriend TL, Elliott ME, Catt KJ. Angiotensin receptors and their antagonists. New Engl J Med 1996; 334:1 649-1654.

3. Pfeffer MA, Braunwald E, Moye LA, Basta L, Brown EJ Jr, Davis $\mathrm{BR}$, et al. Effect of captopril on mortality and morbidity in patients with left ventricular dysfunction after myocardial infarction. Results of the Survival and Ventricular Enlargement Trial. N Engl J Med 1992; 327: 669-677. 
4. The Consensus Trial Study Group: Effects of enalapril on mortality in severe congestive heart failure. N Engl J Med 1987; 316: 1429-1435.

5. Lewis EJ, Hunsicker LG, Bain RP, Rohde RD for the Collaborative Study Group, a clinical trial of an angiotensin converting enzyme inhibitor in the nephropathy of insulin-dependent diabetes mellitus. N Engl J Med 1993; 329: 1456-1462.

6. Hansson L, Lindholm LH, Niskanen L, Lanke J, Hedner T, Niklason A, et al. Effect of angiotensin-converting-enzyme inhibition compared with conventional therapy on cardiovascular morbidity and mortality in hypertension: The Captopril Prevention Project (CAPPP) randomised trial. Lancet 1999; 353: 611-616.

7. Pitt B, Segal R, Martinez FA, Meurers F G, Cowley AJ, Thomas I, et al. Randomised trial of losartan versus captopril in patients over 65 with heart failure (Evaluation of losartan in the elderly study, ELITE). Lancet 1997; 349: 747-752.

8. Lewis E, Hunsicker LG, Clarke WR, Berl T, Pons MA, Lewis JB, et al Renoprotective effect of the angiotensin-receptor antagonist irbesartan in patients with nephropathy due to type 2 diabetes. N Engl J Med 2001; 345: 851-860

9. Parving HH, Lehnert H, Bröchner-Mortensen J, Gomis R, Andersen S, Arner $\mathrm{P}$ for the Irbesartan in patients with type 2 diabetes and microalbuminuria study group. N Engl J Med 2001; 345: 870-878.

10. Juncos LI. Inhibidores de la enzima de conversion. En: Rodicio JL, Romero JC, Ruilope LM (Eds.). Madrid. Fundación para el Estudio de las Enfermedades Cardiovasculares. 1993. pp. 611-628.

11. McInnes GT. Angiotensin II Antagonists. Br J Cardiol 1997; 4 (7): 273 282.

12. Smith DHG, Neutel JM, Morgestern P. Once-daily telmisartan compared with enalapril in the treatment of hypertension. Adv Ther 1998; 15: 229-240.

13. Gradman AH, Arcuri KE, Goldberg AI, Ikeda LS, Nelson EB, Snavely $\mathrm{DB}$, et al. A randomized, placebo-controlled, double-blind, parallel study of various doses of losartan potassium compared with enalapril maleate in patients with essential hypertension. Hypertension 1995; 25: 1345-1350.

14. Larochelle P, Flack JM, Marbury TC, Sarely P, Krieger EM, Reeves RA. Effects and tolerability of irbesartan versus enalapril in patients with severe hypertension. Irbesartan Multicenter Investigators. Am J
Cardiol 1997; 80: 1613-1615.

15. Mimram A, Ruilope L, Kerwin L, Nys M, Owens D, Kassler-Taub K, et al. A randomized, double-blind comparison of the angiotensin II receptor antagonist, irbesartan, with the full dose range of enalapril for the treatment of mild-to-moderate hypertension. J Hum Hypertens 1998; 12: 203-208.

16. Safar ME. Arteries in clinical hypertension. Philadelphia. LippincotRaven Publishers. 1996.

17. Feener EP, King GL. Vascular disfunction in diabetes mellitus. Lancet 1997; 350 (Supl. 1): 9-13.

18. Ruff D, Gazdick LP, Berman R, Goldberg AJ, Sweet CS. Comparative effect of combination drugs therapy regimens commencing with either losartan potassium, an angiotensin II receptor antagonist, or enalapril maleate for the treatment of severe hypertension. J Hypertens 1996; 14: 263-270.

19. Holwerda NJ, Fogari R, Angeli P, Porcellati C, Hereng C, Oddou-Stock $\mathrm{P}$, et al. Valsartan, a new angiotensin II antagonist for the treatment of essential hypertension: eficacy and safety compared with placebo and enalapril. J Hypertens 1996; 14: 1147-1151.

20. Black HR, Graff A, Shutte D, Stoltz R, Ruff D, Levine J, et al. Valsartan, a new angiotensin II antagonist for the treatment of essential hypertension: efficacy, tolerability and safety compared to an angiotensinconverting enzyme inhibitor, lisinopril. J Human Hypertens 1997; 11: 483-489.

21. Bremmer AD, Baur M. Oddou-Stock P, Bodin F. Valsartan: long-term efficacy and tolerability compared to lisinopril in elderly patients with essential hypertension. Clin Exp Hypertens 1997; 19: 1263-1285.

22. Shusterman NH. Safety and efficacy of eprosartan, a new angiotensin II receptor blocker. Am Heart J 1999; 138: S238-S245.

23. Safar ME, Laurent S, Bouthier JD, London GM, Mimran A. Effect of converting enzyme inhibition on hypertension large arteries in humans. Am J Hypertens 1986; 8: 1257-1261.

24. Levy BI, Benessiano J, Poitevin P, Safar ME. Endothelium dependent mechanical properties of the carotid artery in WKY and SHR: role of angiotensin converting enzyme inhibition. Circ Res 1990; 66: 321-328.

25. Dzau VJ, Mukoyama M, Pratt RE. Molecular biology of angiotensin receptors:target for drug research? J Hypertens 1994; 12 (supl 2): S1S5. 